\title{
Bile acid aspiration in people with cystic fibrosis before and after lung transplantation
}

\section{To the Editor:}

Cystic fibrosis (CF) is a genetic condition that is caused by abnormalities in the CF transmembrane conductance regulator (CFTR) gene. People with CF experience life-long morbidity and premature mortality, the vast majority of which is associated with lung disease.

Gastro-oesophageal reflux is known to occur frequently in children and adults with CF, and estimates of prevalence range from $55 \%$ to $90 \%$ [1]. The presence of reflux-induced cough and reported gastro-oesophageal reflux have both been associated with reduced lung function [1]. High levels of pepsin, a gastric protease, have been described in bronchoalveolar lavage (BAL) samples from children with CF [2]. People with CF are predisposed to reflux as a result of both primary and secondary mechanisms and abnormal gastrointestinal motility, with reflux of duodenal contents back into the stomach being also common $[1,3]$. Consistent with these observations, high concentrations of bile acids have been described in the saliva and sputum of both adults and children with CF $[1,4]$.

Reflux is also a common finding in lung transplant recipients [5] and microaspiration of refluxate has been implicated in the pathogenesis of bronchiolitis obliterans syndrome (BOS) [6]. BLONDEAU et al. [1] found bile acids in $60 \%$ of BAL samples from people with CF who had undergone lung transplantation in addition to a series of other studies from the Leuven group indicating a potential role for bile acid aspiration in the pathophysiology of BOS.

We have previously shown that extraction followed by tandem mass spectrometry are necessary to document the low levels of bile acids produced by dilution effects in human BAL [7]. In pilot work, we detected bile acids in the lower airways of nine people homozygous for Phe508del with advanced CF lung disease at the time of lung transplantation [8]. We subsequently hypothesised that bile acids are present in the lower airways of people with CF before and after lung transplantation. To investigate, we used mass spectrometry to detect and identify bile acids in lower airway samples, and oesophageal $\mathrm{pH}$ impedance studies to provide physiological measurements of reflux.

Approval was obtained from Newcastle and North Tyneside 2 and County Durham and Tees Valley 2 Research Ethics Committees respectively. Participants underwent bilateral sequential lung transplantation for advanced CF lung disease; none had undergone antireflux surgery and all were maintained on proton pump inhibitors but none were prescribed promotility agents.

In brief, a $2 \times 15-\mathrm{mL}$ lavage was performed on explanted lungs, centrifuged and the supernatant frozen [9]. Post-transplant, a $3 \times 60-\mathrm{mL}$ BAL was performed in the same individuals at a median of 3 months following transplantation [10]. Electrospray ionisation mass spectrometry was used to detect bile acids as described in detail previously; the lower limit of detection was $0.01 \mu \mathrm{mol} \cdot \mathrm{L}^{-1}$ [7].

Combined 24-h ambulatory $\mathrm{pH}$ impedance was performed with the Medical Measurement System and Ohmega device (MMS Inc., Dover, NH, USA). The impedance catheter was inserted into the oesophagus using standard techniques to place the end of the catheter at the upper border of the lower oesophageal sphincter and the $\mathrm{pH}$ probe $5 \mathrm{~cm}$ above the upper border of the lower oesophageal sphincter [5].

Bile acids were detected in samples from the explanted lungs of all 19 participants with CF and in all BAL samples from the same individuals after lung transplantation (table 1). Of this group, nine out of 19 had oesophageal $\mathrm{pH}$ impedance studies performed post-transplant. These studies identified abnormal overall reflux in seven $(78 \%)$ patients with proximal reflux in four (44\%). In the seven patients with detectable reflux, it was both acid and weakly acid in six, and weakly acid alone in one patient. By comparison, bile acids, including lithocholate, were largely undetectable in BAL samples from six non-CF patients post-lung transplant (data not shown).

These results confirm that bile acids are present in the lower airways of people with advanced CF lung disease, confirming previous observations restricted to Phe508del homozygotes [8]. Longitudinal follow-up showed that bile acids continue to be detectable in the lower airways post-lung transplantation. Furthermore, we found abnormal levels of reflux in most people with CF tested post-transplant. This rare 
TABLE 1 Brief clinical details and bile acid concentrations pre- and post-lung transplantation measured by electrospray ionisation mass spectrometry

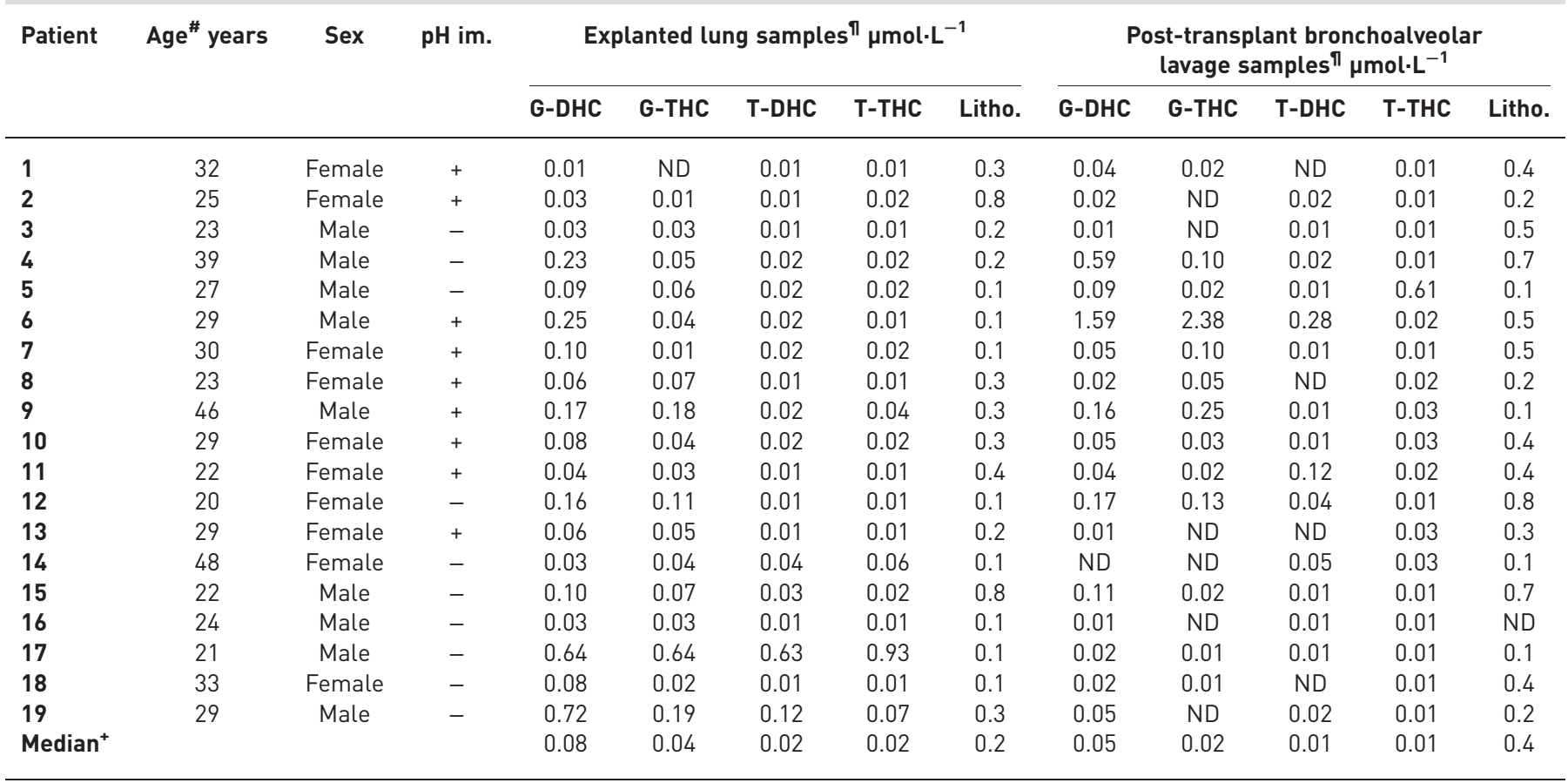

pH im.: pH impedance; G-DHC: glycodihydroxycholanoate (deoxycholate and isomers, glycine conjugate); G-THC: glycotrihydroxycholanoate (cholate and isomers, glycine conjugate); T-DHC: taurodihydroxycholanoate (deoxycholate and isomers, taurine conjugate); T-THC: taurotrihydroxycholanoate (cholate and isomers, taurine conjugate); Litho.: unconjugated lithocholate; +: performed; -: not performed; ND: not detected (less than lower limit of detection $0.01 \mu \mathrm{mol} \cdot \mathrm{L}^{-1}$ )..$^{\#}$ : at time of transplant (complete years only); ${ }^{\text {?: }}$ : raw data of bile acids detected in 180 -mL bronchoalveolar lavages. ${ }^{+}:$for bile acids where the level was below the limit of detection, the median was calculated after nondetectable samples were assigned an arbitrary low value of $0.01 \mu$ mol. $L^{-1}$.

longitudinal information indicates that reflux and aspiration are not simply a function of factors associated with chronic lung disease; we demonstrate persistence post-transplantation, where cough is attenuated while thoracic mechanical changes caused by advanced lung disease are corrected.

We used mass spectrometric bile assays, suitable for the low levels found in BAL samples, providing specific information about individual bile acids [7]. There are no studies characterising individual aspirated bile acids in the CF lung that we are aware of. We measured median lithocholate levels of 0.2 and $0.4 \mu \mathrm{mol} \cdot \mathrm{L}^{-1}$ pre- and post-lung transplantation, respectively. Lithocholic acid was recently detected following direct tracheal suction at a mean \pm SEM concentration of $2.8 \pm 0.6 \mu \mathrm{mol} \cdot \mathrm{L}^{-1}$ in critically unwell patients with ventilator-associated pneumonia [11]. Elevated levels of bile acids were associated with lung injury and were raised compared to ventilated patients with no pneumonia. Furthermore, $\mathrm{Wu}$ et al. [11] showed that chenodeoxycholic acid challenge of alveolar epithelial cells led to interleukin-8 generation.

Lithocholate is normally a minor component of circulating bile acids, produced by specific gut bacteria that deconjugate and dehydroxylate the primary bile acid chenodeoxycholate. Bile acid homeostasis and metabolism may be disordered in CF [12]. CF is a multisystem disorder that involves both the respiratory and gastrointestinal tracts. Duodeno-gastro-oesophageal reflux and subsequent microaspiration of bile acids may be an under-recognised contributor to airway injury in CF lung disease [13]. Treatments for reflux exist and a very limited literature has advocated fundoplication in CF. In a recent small open study of adult CF patients there was a fall in cough, and exacerbation events reduced by $50 \%$ post-fundoplication [13]. Fundoplication in children with CF has also been undertaken but results have been varied [14].

It is important that we acknowledge the limitations of our study. For technical reasons, a small-volume lavage was performed in the noninflated resected lungs compared to the $180-\mathrm{mL}$ BAL used to sample allografts. There is still no entirely satisfactory method of determining the dilution factor during BAL.

Post-transplantation, people with CF have comparable levels of BOS to patients transplanted for other indications. However, the post-transplantation CF population is heterogeneous and some individuals develop BOS with an aggressive onset; this is now recognised as a complex, alloimmune and non-alloimmune response to injury. Colonisation of CF allografts with Pseudomonas aeruginosa has been associated with bile aspiration and neutrophilic airway inflammation by the Leuven group [15]. In turn, infection and 
inflammation, associated with bile aspiration $[11,15]$, have been linked to BOS and death in 260 lung transplant recipients in a recent series [16]. Further studies are therefore required in greater numbers of patients. There is potential that outcomes of lung transplantation in CF, already comparable with other indications on average, could be further improved if aspiration injury were reliably detected and treated.

In conclusion, we have shown that bile acids measured by tandem mass spectrometry are detectable in the lower airway in advanced CF lung disease and that this potential source of injury persists after lung transplantation. Interactions between gastrointestinal and lung pathophysiologies may contribute to overall lung damage and this "aerodigestive" concept may have therapeutic implications in a disease where available treatments are otherwise limited. transplantation http://ow.ly/RTvNW

Malcolm Brodlie $^{1,2}$, Ali Aseeri ${ }^{1,3}$, Jim L. Lordan ${ }^{1,4}$, Andrew G.N. Robertson ${ }^{5}$, Michael C. McKean ${ }^{2}$, Paul A. Corris ${ }^{1,4}$, S. Michael Griffin ${ }^{5}$, Nigel J. Manning ${ }^{6}$, Jeffrey P. Pearson ${ }^{3}$ and Christopher Ward ${ }^{1}$

${ }^{1}$ Institute of Cellular Medicine, Newcastle University, Newcastle upon Tyne, UK. ${ }^{2}$ Department of Paediatric Respiratory Medicine, Great North Children's Hospital, Newcastle upon Tyne Hospitals NHS Foundation Trust, Newcastle upon Tyne, UK. ${ }^{3}$ Institute for Cell and Molecular Bioscience, Newcastle University, Newcastle upon Tyne, UK. ${ }^{4}$ Institute of Transplantation, Freeman Hospital, Newcastle upon Tyne Hospitals NHS Foundation Trust, Newcastle upon Tyne, UK. ${ }^{5}$ Northern Oesophago-Gastric Unit, Royal Victoria Infirmary, Newcastle upon Tyne Hospitals NHS Foundation Trust, Newcastle upon Tyne, UK. 'Department of Clinical Chemistry, Sheffield Children's Hospital, The Sheffield Children's NHS Trust, Sheffield, UK.

Correspondence: Chris Ward, Room 1.072, Catherine Cookson Building, Medical School, Newcastle University, Framlington Place, Newcastle upon Tyne, NE2 4HH, UK. E-mail: chris.ward@ncl.ac.uk

Received: March 312015 | Accepted after revision: Aug 122015 | First published online: Oct 222015

Support statement: Funding for this work was gratefully received from a Saudi Arabian government studentship (A. Aseeri, J.P. Pearson and C. Ward), Medical Research Council (MRC)/CF Trust Clinical Research Training and Clinician Scientist Fellowships (M. Brodlie), an MRC Project Grant (C. Ward and P.A. Corris), the Newcastle upon Tyne Hospitals NHS Foundation Trust Special Trustees (M.C. McKean, M. Brodlie, C. Ward and J.L. Lordan) and the British Lung Foundation Trevor Clay Memorial Award (C. Ward, J.P. Pearson and A.G.N. Robinson). Funding information for this article has been deposited with FundRef.

Conflict of interest: None declared.

Acknowledgements: We gratefully acknowledge the help of the patients involved in this study along with all members of the wider cardiothoracic transplantation team at the Freeman Hospital.

\section{References}

1 Blondeau K, Dupont LJ, Mertens V, et al. Gastro-oesophageal reflux and aspiration of gastric contents in adult patients with cystic fibrosis. Gut 2008; 57: 1049-1055.

2 McNally P, Ervine E, Shields MD, et al. High concentrations of pepsin in bronchoalveolar lavage fluid from children with cystic fibrosis are associated with high interleukin-8 concentrations. Thorax 2011; 66: 140-143.

3 Pauwels A, Blondeau K, Mertens V, et al. Gastric emptying and different types of reflux in adult patients with cystic fibrosis. Aliment Pharmacol Ther 2011; 34: 799-807.

4 Pauwels A, Decraene A, Blondeau K, et al. Bile acids in sputum and increased airway inflammation in patients with cystic fibrosis. Chest 2012; 141: 1568-1574.

5 Robertson AG, Krishnan A, Ward C, et al. Anti-reflux surgery in lung transplant recipients: outcomes and effects on quality of life. Eur Respir J 2012; 39: 691-697.

6 Stovold R, Forrest IA, Corris PA, et al. Pepsin, a biomarker of gastric aspiration in lung allografts: a putative association with rejection. Am J Respir Crit Care Med 2007; 175: 1298-1303.

7 Parikh S, Brownlee IA, Robertson AG, et al. Are the enzymatic methods currently being used to measure bronchoalveolar lavage bile salt levels fit for purpose? J Heart Lung Transplant 2013; 32: 418-423.

8 Aseeri A, Brodlie M, Lordan J, et al. Bile acids are present in the lower airways of people with cystic fibrosis. Am J Respir Crit Care Med 2012; 185: 463.

9 Brodlie M, McKean MC, Johnson GE, et al. Raised interleukin-17 is immunolocalised to neutrophils in cystic fibrosis lung disease. Eur Respir J 2011; 37: 1378-1385.

10 Ward C, Eger K, Diboll J, et al. Bronchial epithelial cells cultured from clinically stable lung allograft patients promote the development of macrophages from monocytes rather than dendritic cells. Thorax 2009; 64: 430-435.

11 Wu YC, Hsu PK, Su KC, et al. Bile acid aspiration in suspected ventilator-associated pneumonia. Chest 2009; 136: $118-124$.

12 Debray D, Rainteau D, Barbu V, et al. Defects in gallbladder emptying and bile acid homeostasis in mice with cystic fibrosis transmembrane conductance regulator deficiencies. Gastroenterology 2012; 142: 1581-1591.

13 Fathi H, Moon T, Donaldson J, et al. Cough in adult cystic fibrosis: diagnosis and response to fundoplication. Cough 2009; 5: 1.

14 Boesch RP, Acton JD. Outcomes of fundoplication in children with cystic fibrosis. J Pediatr Surg 2007; 42: 1341-1344. 
15 Vos R, Blondeau K, Vanaudenaerde BM, et al. Airway colonization and gastric aspiration after lung transplantation: do birds of a feather flock together? J Heart Lung Transplant 2008; 27: 843-849.

16 Gregson AL, Wang X, Weigt SS, et al. Interaction between Pseudomonas and CXC chemokines increases risk of bronchiolitis obliterans syndrome and death in lung transplantation. Am J Respir Crit Care Med 2013; 187: $518-526$

\section{Chronic pulmonary disease with Mycobacterium abscessus complex is a biofilm infection}

To the Editor:

Direct evidence of Mycobacterium abscessus complex (MABSC) biofilm in the human lung has not previously been demonstrated. Biofilms are microcolonies of bacteria, imbedded in extracellular matrix, providing stability and tolerance to antibiotics and the body's innate and adaptive defences [1]. This mode of growth is an inherent feature of chronic infections and is particularly well studied for Pseudomonas aeruginosa and other Gram-negative infections [1], but also some Staphylococcus aureus infections [2]. Mycobacterial infections have also been shown to be capable of biofilm formation, most notably Mycobacterium tuberculosis (tuberculosis), which under the right conditions, can self-assemble into highly organised matrix-encapsulated biofilm [3]. Among the nontuberculous mycobacteria (NTM), Mycobacterium avium complex (MAC) and the rapidly growing mycobacteria, including MABSC, have been shown to grow as biofilms either in vitro or in environmental reservoirs $[4,5]$, but in vivo conditions have not been studied. MABSC is an emerging threat to patients with cystic fibrosis [6], who become infected at an early age and deteriorate clinically [7] as the persistent infection causes inflammation and tissue damage. We wanted to explore how MABSC grows in the antibiotic-rich, end-stage lungs of patients with cystic fibrosis. The aim was to describe the localisation and growth patterns of MABSC in vivo from freshly explanted lungs of patients with cystic fibrosis and a history of MASBC. We simultaneously performed histological and mycobacterial sampling from the same areas, from multiple pulmonary sites. Mycobacterial culture was performed by inoculation on at least one solid (Middlebrooke $7 \mathrm{H} 10$ or Löwenstein-Jensen slopes; SSI Diagnostica, Hilleroed, Denmark) and in one liquid culture medium (BACTEC 12B or MGIT; Becton Dickinson Microbiology Systems, Sparks, MD, USA), and a reverse hybridisation DNA assay was performed (InnoLiPA; Fujirebio Europe, Brondby, Denmark), as previously described [7]. Culture morphology was determined by direct visual inspection of colonies and control microscopy used Ziehl-Neelsen staining. Concomitant culture for Gram-negative and -positive bacteria was also performed. Patient files were reviewed to determine the clinical course of their end-stage lung disease and their history of other chronic bacterial infections. The NTM collection and clinical data collection was approved by the Committee on Health Research Ethics in the Capital Region of Denmark (Copenhagen, Denmark) (H-3-2012-098). The explanted lungs were collected by the transplantation team, microbiological samples were sent for NTM culture and histological samples were transferred to $4 \%$ formaldehyde before further preparation for microscopic investigation. The biopsy material was embedded in paraffin, cut into 4- $\mu \mathrm{m}$ sections and mounted on glass slides. Prior to microscopy, paraffin was removed and the tissue sections were analysed by means of conventional haematoxylin and eosin (H\&E) staining, Ziehl-Neelsen staining (acid-fast stain), auramine dye (fluorescent stain), and fluorescent in situ hybridisation (FISH) with peptide nucleic acid (PNA) probes: a uniBac probe (red) and a NTM-specific probe (green) (AdvanDx, Inc., Woburn, MA, USA). We used 4',6'-diamidino-2-phenylindole (Vector Laboratories) as a counterstain for DNA. Microscopic observations were performed with Zeiss 710 Confocal Laser Scanning Microscope (Leica Microsystems, Mannheim, Germany).

Six patients with either active $(n=5)$ or prior $(n=1)$ MABSC infection were included. None had a history of infection with other NTM species. The five patients with active MABSC disease were in intravenous or oral multidrug antimycobacterial treatment at the time of transplantation. Their clinical outcomes have previously been published [8]. MABSC was cultured from the lungs of all five patients with ongoing infection, three of which had MABSC smooth colony morphology. Morphology data was not recorded for 